\title{
Imaging of NGC 5907's stellar stream
}

\author{
Seppo Laine ${ }^{1}$, Carl J. Grillmair ${ }^{1}$, David Martínez-Delgado², \\ Aaron J. Romanowsky ${ }^{3}$, Peter L. Capak ${ }^{1}$, Richard G. Arendt ${ }^{4}$, \\ Matthew L. N. Ashby ${ }^{5}$, James E. Davies ${ }^{6}$, Steven R. Majewski ${ }^{7}$ \\ and R. Jay GaBany ${ }^{8}$ \\ ${ }^{1}$ Spitzer Science Center-Caltech, MS 314-6, Pasadena, CA 91125, USA, email: \\ seppo@ipac.caltech.edu ${ }^{2}$ Astronomisches Rechen-Institut, Zentrum für Astronomie der \\ Universität Heidelberg, Mönchhofstr. 12-14, D-69120 Heidelberg, Germany, ${ }^{3}$ Department of \\ Physics and Astronomy, San José State University, One Washington Square, San Jose, \\ CA 95192, ${ }^{4}$ CRESST/UMBC/NASA GSFC, Code 665, Greenbelt, MD 20771, USA, \\ ${ }^{5}$ Harvard-Smithsonian Center for Astrophysics, 60 Garden St., Cambridge, MA 02138, USA, \\ ${ }^{6}$ Minor Planet Center, Harvard-Smithsonian Center for Astrophysics, 60 Garden St, MS-18, \\ Cambridge, MA 02138. USA, ${ }^{7}$ Department of Astronomy, University of Virginia, \\ Charlottesville, VA 22904, USA, ${ }^{8}$ Black Bird Observatory, 5660 Brionne Drive, San Jose, \\ CA 95118, USA
}

\begin{abstract}
We have obtained deep $g, r$, and $i$-band Subaru and ultra-deep $3.6 \mu \mathrm{m} \mathrm{IRAC} \mathrm{images}$ of parts of the multiply-wrapped stellar stream around the nearby edge-on galaxy NGC 5907 . We have fitted the surface brightness measurements of the stream with FSPS stellar population synthesis models to derive the metallicity and age of the brightest parts of the stream. The resulting relatively high metallicity $([\mathrm{Fe} / \mathrm{H}]=-0.3)$ is consistent with a major merger scenario but a satellite accretion event cannot be ruled out.
\end{abstract}

Keywords. galaxies: evolution, galaxies: halos, galaxies: individual (NGC 5907), galaxies: interactions, galaxies: structure

\section{Introduction}

The detection of stellar streams around nearby galaxies (e.g., Martínez-Delgado et al. 2008, 2009, 2010) provides unequivocal evidence of galaxy accretion events. These features reveal the mechanism e.g. for the hierarchical growth of galaxy halos. However, little is known about the progenitors of stellar streams, including their mass and metallicity. While most of the evidence of minor interactions has come from observations of the Milky Way and M31, nearby galaxies outside the Local Group provide even more spectacular evidence of such galaxy disruption events. In this contribution we examine the multiply-wrapped stellar stream around the nearby $(D \approx 17 \mathrm{Mpc})$ edge-on disk galaxy NGC 5907 with visible light and near-infrared observations.

\section{Observations, Data Reduction and Results}

NGC 5907 was observed with Subaru's Suprime-Cam imager in $g$-, $r$-, and $i$-bands, and with Spitzer's IRAC camera at $3.6 \mu \mathrm{m}$. We used an iterative scheme to subtract the background light in Subaru images. We ran the IRAC data through Fixen selfcalibration (Fixen et al. 2000) and through one iteration of the GOODS pipeline (Grumm et al. 2005) artifact mitigation and then mosaicked the frames. We masked the foreground and background sources by hand before measuring the surface brightness along the brightest part of the stream. We fitted the surface brightnesses with FSPS SED models from Conroy et al. (2009) and Conroy \& Gunn (2010). The best fit (metallicity 


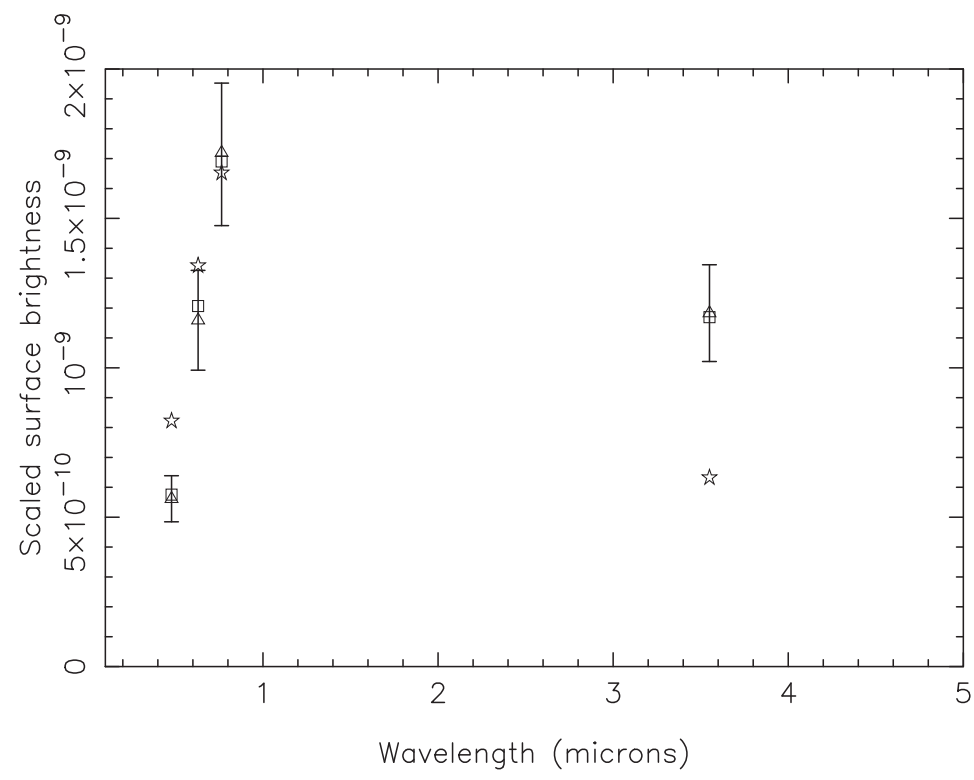

Figure 1. Observations (triangles), best-fit model (squares) and a low metallicity model (stars) in the brightest part of the stellar stream of NGC 5907.

$[\mathrm{Fe} / \mathrm{H}]=-0.3$, age $=14.96 \mathrm{Gyrs})$ is shown in Figure 1 where we also show a model with a low metallicity $([\mathrm{Fe} / \mathrm{H}]=-1.98$, age $=9.44$ Gyrs $)$.

Martínez-Delgado et al. (2008) modeled the stellar stream with a satellite companion having a stellar mass of $\approx 3.5 \times 10^{8} \mathrm{M}_{\odot}$. In contrast, Wang et al. (2012) modeled the stream with a major merger (mass ratio $1: 3-1: 12$ ). While our relatively high metallicity value suggests a major merger origin of the stream, it is possible that the metallicity is consistent with a satellite accretion event such as the Sagittarius Stream (Chou et al. 2007) or the giant stream in M31 (Kalirai et al. 2006). This work is based in part on observations made with the Spitzer Space Telescope, operated by JPL/Caltech under a contract with NASA and with the Subaru Telescope, operated by NAOJ. Support for this work was provided by NASA through an award issued by JPL/Caltech.

\section{References}

Chou, M.-Y., et al. 2007, ApJ, 670, 346

Conroy, C., Gunn, J. E., \& White, M. 2009, ApJ, 699, 486

Conroy, C. \& Gunn, J. E. 2010, ApJ, 712, 833

Fixen, D. J., Moseley, S. H., \& Arendt, R. G. 2000, ApJS, 128, 651

Grumm, D., Casertano, S., Dickinson, M., \& Holfeltz, S. 2005, in: P. Shopbell, M. Britton \& R. Ebert (eds.) ASP-CS 347, Astronomical Data Analysis Software and Systems XIV, (San Francisco: ASP), p. 454

Kalirai, J., et al. 2006, ApJ, 641, 268

Martínez-Delgado, D., Peñarrubia, J., Gabany, R. J., Trujillo, I., Majewski, S. R., \& Pohlen, M. 2008, ApJ, 689, 184

Martínez-Delgado, D., Pohlen, M., Gabany, R. J., Majewski, S. R., Peñarrubia, J., \& Palma, C. $2009, A p J, 692,955$

Martínez-Delgado, D., et al. 2010, AJ, 140, 962

Wang, J., Hammer, F., Athanassoula, E., Puech, M., Yang, Y., \& Flores, H. 2012, A\&্A, 538, A121 